\title{
Freak Descriptor With Spatial Pyramid Kernel For Scene Categorization
}

\author{
Qiong Yao \\ School of Computer Engineering \\ University of Electronic Science and Technology \\ ZhongShan, China \\ 2542807@qq.com
}

\author{
Xiang Xu \\ School of Computer Engineering \\ University of Electronic Science and Technology \\ ZhongShan, China \\ 467796538@qq.com
}

\begin{abstract}
Spatial Pyramid Kernel performs good on challenging scene categorization tasks for combining multi-resolution and spatial information of image features, while the popular feature extraction still be the global SIFT or GIST, neglecting more recent, effective and efficient descriptors, such as FREAK, which is faster to compute , more compact, and with lower memory. We partition image into fixed sub-regions, compute dense freak descriptor over fixed subregions' center points instead of on interest points, then use the spatial pyramid kernel recognition method for scene categorization. We make two comparisons, the first is between the FREAK (Fast Retina Keypoint) and the SIFT descriptor under the same scene categorization framework and the second is compute dense freak descriptors over fixed subregions' center points versus on interest points. The results show that the dense freak descriptor ensures a very competitive categorization performance with lower computational cost and less memory. It indicates that the FREAK descriptor is avaiable alternative to the SIFT descriptor for the problem of scene categorization and particularly useful for real-time-recognition systems for low-resource devices such as mobile phones.
\end{abstract}

Keywords-Spatial Pyramid Kernel; FREAK; Sift; Scene Categorization; SVM

\section{I . INTRODUCTION}

The performance of scene categorization system depends mainly on two components. First, a suitable bag-of-features representation and second a powerful SVM kernel[10] on this representation. Despite the fact that a lot of advancements have been made in the area of keypoint descriptors over the last years, the literature on scene categorization for the most part still focuses on established descriptors, such as Lowe's SIFT [1] and torralba's "GIST" [2] descriptors, and largely neglects more recent descriptors, such as the FREAK descriptor [3] which is faster to compute, more compact while remaining robust to scale, rotation and noise. On the other hand , SVMs [9] are standard techniques in machine learning and excel by their ability to control the regularization, but they need a suitable kernel in order to work well. The spatial pyramid kernel [4] works perfect by computing rough geometric correspondence on a global scale using an efficient approximation technique adapted from the pyramid matching scheme of Grauman and Darrell[5], the recognizing scheme involves repeatedly subdividing the image and computing histograms of local features at increasingly fine resolutions.

We partition image into fixed sub-regions, compute dense freak descriptor over fixed subregions' center points instead of on interest points, then use the spatial pyramid kernel recognition method for scene categorization. We make two comparisions, the first is between the FREAK (Fast Retina Keypoint) and the SIFT descriptor under the same scene categorization framework and the second is compute dense freak descriptors over fixed subregions' center points versus on interest points. The results show that the dense freak descriptor ensures a very competitive categorization performance with lower computational cost and less storge space. It indicates that the FREAK descriptor is avaiable alternative to the SIFT descriptor for the problem of scene categorization and particularly useful for real-time-recognition systems for low-resource devices such as mobile phones.

In this paper we try to make a experimental comparision between the dense SIFT and dense FREAK desctiptors over subregions' center points instead of on interest points for the task of scene categorization. We use the spatial pyramid matching framwork presented in[4], and apply the framework to images databases from the fifteeen scenes[6] and the caltech101[7]. The results of our experiments suggest that the FREAK descriptor represents an appealing alternative to the SIFT descriptor with lower computational cost and storage space.

The rest of the paper is structured as follows. In Section II we introduce the Spatial Pyramid kernel. In Sections III we briefly describe the theory of the FREAK descriptors. In Section IV we present the experimental results and our main findings. We conclude the paper with some final comments and direction for future work in section $\mathrm{V}$.

\section{II . SPATIAL Pyramid KeRNEL}

Grauman and Darrell [5] propose pyramid matching kernel to find an approximate correspondence between two sets. Let $\mathrm{X}$ and $\mathrm{Y}$ be two sets of vectors in a d-dimensional 
feature space, construct a sequence of grids at resolutions $0, \cdots, L$, such that the grid at level $l$ has $2^{l}$ cells along each dimension, for a total of $D=2^{d l}$ cells. Let $H_{X}^{l}$ and $H_{Y}^{l}$ denote the histograms of $X$ and $Y$ at this resolution, so that $H_{X}^{l}(i)$ and $H_{Y}^{l}(i)$ are the numbers of points from $X$ and $Y$ that fall into the $i$ th cell of the grid. Then the number of matches at level $l$ is given by the histogram intersection function:

$$
I\left(H_{X}^{l}, H_{Y}^{l}\right)=\sum_{i=1}^{D} \min \left(H_{X}^{l}(i), H_{Y}^{l}(i)\right)
$$

The weight associated with level $l$ is set to $\frac{1}{2^{L-l}}$

,which is inversely proportional to cell width at that level, and penalizing matches found in larger cells because they involve increasing dissimilar features, the pyramid match kernel is definied by :

$$
\begin{aligned}
k^{L}(X, Y) & =I^{L}+\sum_{l=0}^{L-1} \frac{1}{2^{L-l}}\left(I^{l}-I^{l+1}\right) \\
& =\frac{1}{2^{L}} I^{0}+\sum_{l=1}^{L} \frac{1}{2^{L-l+1}} I^{l}
\end{aligned}
$$

Then quantizing all feature vectors into $\mathrm{M}$ discrete types, and make the simplifying assumption that only features of the same type can be matched to one another. Each channel $m$ gives us two sets of two-dimensional vectors, $X_{m}$ and $Y_{m}$ representing the coordinates of features of type $m$ found in the respective images. The final kernel is then the sum of the separate channel kernels:

$$
K^{L}(X, Y)=\sum_{m=1}^{M} k^{L}\left(X_{m}, Y_{m}\right)
$$

\section{FREAK DESCRIPTOR}

\section{A. Retinal sampling pattern}

The sampling pattern adopted by the FREAK approach is biologically inspired by the retinal pattern in the eye. Before the descriptor is computed, the $\mathrm{N}$ sample points located around the given keypoints are smoothed with a Gaussian kernel. Here, the size of the kernel is varied with respect to the location of the sampling point to simulate the behavior of the human retina. In analogy to the human visual system, the sampling points of the FREAK descriptor, hence , represent the centers of the receptive fields.
Mathematically, this can be defined as follows:

$$
P_{i}=P\left(x_{i}, y_{i}\right)=L_{r_{i}}\left(x_{i}, y_{i}\right)
$$

Where

$$
L_{r_{i}}(x, y)=I(x, y) * G_{r_{i}}\left(x, y, \sigma_{r_{i}}\right)
$$

In the above equations $I(x, y)$ stands for the input image, $G_{r_{i}}\left(x, y, \sigma_{r_{i}}\right)$ denotes the Gaussian kernel for the $i_{\text {-th receptive field }(i=1,2, \cdots, N}$ ) and $L_{r_{i}}(x, y)$ represents the smoothed version of the input image. The $i_{\text {-th sampling point }} P_{i}$ correspoinding to the center of the $i_{\text {-th receptive field }} r_{i}$ and is defined with the predefined coordinates $\left(x_{i}, y_{i}\right)$ from the sampling pattern, where $i=1,2, \cdots, N$.

\section{B. Building the descriptor}

$P_{a}=\left(P_{i}, P_{j}\right)$, where $i, j \in\{1,2, \cdots, N\}$ and $i \neq j$ the FREAK approach defines a binary encoded intensity comparision $s\left(P_{a}\right)$ on this pair as

$$
s\left(P_{a}\right)= \begin{cases}1 & \text { if } P_{i}>P_{j} \\ 0 & \text { otherwise }\end{cases}
$$

The presented comparision forms the basis for building the FREAK descriptor $\mathrm{F}$ as a $\mathrm{N}$-dimensional bit string:

$$
F=\sum_{0 \leq a<N} 2^{a} s\left(P_{a}\right)
$$

\section{C.Orientation normalization}

The orientation of the FREAK descriptor is estimated based on 45 selected sampling-point pairs that are arranged symmetrically with respect to the center of the sampling pattern. Let $G$ be the set of all the selected pairs and assume that local gradients have been computed for all the selected sampling points, then the orientation $O$ of the given keypoint can be computed as:

$$
o=\frac{1}{M} \sum_{P_{i}, P_{j} \in G}\left(P_{i}-P_{j}\right) \frac{T\left(P_{i}\right)-T\left(P_{j}\right)}{\left\|T\left(P_{i}\right)-T\left(P_{j}\right)\right\|}
$$

Where $M$ is the number of pairs in $G$ and

$T\left(P_{i}\right)$ denotes a function returning the $2 \mathrm{D}$ vector of the spatial coordinates of the center of receptive field, i.e. the vector of coordinates of the $\mathrm{k}$-th sampling point $T\left(P_{k}\right)=\left[x_{k}, y_{k}\right]$.

\section{EXPERIMENTS}

In this section, three comparision experiments are carried out . First, extract dense SIFT descriptors of 
$16 \times 16$ pixel patches computed over a grid with spacing of 8 pixels, second, extract dense freak descriptors on the same grid, third, extract freak descriptors on the interest points[8]. We follow the experimental setup of [4], Typical vocabulary sizes are $M=200$, set pyramid levels are $\mathrm{L}=2$. The experiment image databases are fifteen scene categories[6] and Caltech-101[7]. Our first dataset is composed of fifteen scene categories[6], each category has 200 to 400 images, and average image size is $300 \times 250$ pixels. The second set of experiments is on the Caltech-101[7],this database contains from 31 to 800 images per category. Most images are medium resolution,i.e., about $300 \times 300$ pixels.

TABLE I THE MEAN AND STANDARD DEVIATION OF CALSSIFICATION RESULTS FOR THE DATABASES

\begin{tabular}{|l|l|l|l|}
\hline $\begin{array}{l}\text { Image } \\
\text { database }\end{array}$ & $\begin{array}{l}\text { Dense SIFT } \\
\text { features(\%) }\end{array}$ & $\begin{array}{l}\text { Dense Freak } \\
\text { features(\%) }\end{array}$ & $\begin{array}{l}\text { Freak on } \\
\text { keypoint(\%) }\end{array}$ \\
\hline 15 scene & $92.52 \pm 1.09$ & $94.16 \pm 0.57$ & $48.67 \pm 1.55$ \\
\hline Caltech101 & $91.85 \pm 1.11$ & $93.99 \pm 0.58$ & $46.53 \pm 1.58$ \\
\hline
\end{tabular}

TABLE II AVERAGE CLASSIFICATION RATES FOR INDIVIDUAL CLASSES ON THE FIFTEEN SCENE DATABASE

\begin{tabular}{|l|l|l|l|}
\hline Classes & $\begin{array}{l}\text { Dense SIFT } \\
\text { features(\%) }\end{array}$ & $\begin{array}{l}\text { Dense Freak } \\
\text { features(\%) }\end{array}$ & $\begin{array}{l}\text { Freak on } \\
\text { keypoint(\%) }\end{array}$ \\
\hline 01bedroom & 93.52 & 96.76 & 54.63 \\
\hline 02CALsuburb & 99.59 & 99.59 & 74.27 \\
\hline 03industrial & 80.06 & 88.75 & 26.37 \\
\hline 04kitchen & 94.29 & 97.14 & 59.52 \\
\hline 05livingroom & 98.27 & 96.54 & 34.60 \\
\hline 06MITcoast & 80.56 & 92.50 & 46.11 \\
\hline 07MITforest & 98.17 & 100 & 96.04 \\
\hline 08MIThighway & 87.31 & 95.38 & 50.77 \\
\hline 09MITinsidecity & 92.53 & 94.81 & 22.40 \\
\hline 10MITmountain & 88.77 & 91.44 & 37.70 \\
\hline 11MITopencountry & 90.24 & 92.68 & 33.66 \\
\hline 12MITstreet & 97.60 & 93.15 & 70.55 \\
\hline 13MITtallbuilding & 87.36 & 87.08 & 46.35 \\
\hline 14PARoffice & 93.95 & 96.74 & 36.28 \\
\hline 15store & 99.05 & 100 & 80.95 \\
\hline
\end{tabular}

We train on 100 images per class on first database and 30 images per class on second database and test on the rest. All experiments are repeated ten times with different randomly selected training and testing images, the average of per-class recognition rates is recorded for each run. The final result is reported as the mean and standard deviation of the results from the individual runs.
Table I shows detailed results of respective classification results for two databases. Dense freak descriptor performs best, achieve $94.16 \%$ and $93.99 \%$ average classification rate on two image databases respectively, dense sift descriptor achieve $92.52 \%$ and $91.85 \%$ average classification rate on two image databases respectively, freak descriptor on keypoints performs worst ,only achieve $48.67 \%$ and $46.53 \%$ respectively. Table II shows average classification rates for individual classes on the

TABLEIII TOP FIFTEEN LOW AVERAGE CLASSIFICATION RATES FOR INDIVIDUAL CLASSES ON THE CALTECH-101 DATABASE

\begin{tabular}{|c|c|c|c|c|c|}
\hline Classes & $\begin{array}{l}\text { Dense } \\
\text { SIFT }\end{array}$ & Classes & $\begin{array}{l}\text { Dense } \\
\text { Freak }\end{array}$ & Classes & $\begin{array}{l}\text { Freak } \\
\text { on }\end{array}$ \\
\hline & $\begin{array}{l}\text { feature } \\
\text { s(\%) }\end{array}$ & & $\begin{array}{l}\text { feature } \\
\text { s(\%) }\end{array}$ & & $\begin{array}{l}\text { keypoi } \\
\text { nt(\%) }\end{array}$ \\
\hline saxophone & 60 & pigeon & 68.89 & ewer & 27.06 \\
\hline starfish & 61.63 & $\begin{array}{l}\text { BACKGR } \\
\text { OUND_G } \\
\text { oogle }\end{array}$ & 78.37 & Ferry & 28.36 \\
\hline chair & 66.13 & scissors & 79.49 & nautilus & 30.91 \\
\hline scorpion & 67.86 & buddha & 80 & $\begin{array}{l}\text { BACK } \\
\text { GROU } \\
\text { ND_Go } \\
\text { ogle }\end{array}$ & 31.69 \\
\hline butterfly & 68.13 & beaver & 80.43 & $\begin{array}{l}\text { butterfl } \\
\mathrm{y}\end{array}$ & 31.87 \\
\hline pigeon & 71.11 & stop_sign & 81.25 & camera & 32 \\
\hline mandolin & 72.09 & mayfly & 82.50 & $\begin{array}{l}\text { sea_hor } \\
\text { se }\end{array}$ & 33.33 \\
\hline flamingo & 76.12 & menorah & 82.76 & chair & 33.87 \\
\hline gramophone & 76.47 & umbrella & 84 & $\begin{array}{l}\text { strawbe } \\
\text { rry }\end{array}$ & 34.29 \\
\hline strawberry & 77.14 & revolver & 84.15 & $\begin{array}{l}\text { menora } \\
\mathrm{h}\end{array}$ & 34.48 \\
\hline $\begin{array}{l}\text { Electric_guit } \\
\text { ar }\end{array}$ & 78.67 & scorpion & 84.52 & crab & 36.99 \\
\hline laptop & 79.01 & $\begin{array}{l}\text { stegosaur } \\
\text { us }\end{array}$ & 84.75 & $\begin{array}{l}\text { brontos } \\
\text { aurus }\end{array}$ & 37.21 \\
\hline $\begin{array}{l}\text { BACKGRO } \\
\text { UND_Googl } \\
\text { e }\end{array}$ & 79.87 & $\begin{array}{l}\text { ceiling_fa } \\
\mathrm{n}\end{array}$ & 85.11 & $\begin{array}{l}\text { gramop } \\
\text { hone }\end{array}$ & 39.22 \\
\hline beaver & 80.43 & lamp & 86.89 & lotus & 39.39 \\
\hline umbrella & 81.33 & bass & 87.04 & $\begin{array}{l}\text { Dragonf } \\
\text { ly }\end{array}$ & 39.71 \\
\hline
\end{tabular}


fifteen scene database.Table III shows top fifteen low average classification rates for individual classes on the Caltech-101 database.

\section{DISCUSSION}

This paper we repeatedly subdividing an image and computing histograms of dense freak features over the resulting subregions, has shown promising results on two large-scale,diverse datasets compared with dense sift and sparse freak on keypoints. For the FREAK descriptor is faster to compute, more compact, and with lower memory. It indicates that the FREAK descriptor is avaiable alternative to the SIFT descriptor for the problem of scene categorization and particularly useful for real-time-recognition systems for low-resource devices such as mobile phones.

\section{ACKNOWLEDGMENT}

This work was financially supported by the ZhongShan Science and Technology Planning Project (413S40), and Guangdong province science and technology plan projects (2013B090500035).

\section{REFERENCE}

[1] D.G.Lowe. Distinctive image features from scale-invariant keypoints. IJCV,60,2, pp.91-110,2004.

[2] A.Torralba, K.P.Murphy, W.T.Freeman, and M.A.Rubin. Context-based vision system for place and object recognition. ICCV pp.273-280, 2003.

[3] A .Alahi, R. Ortiz, P.Vandergheynst, FREAK:Fast Retina Keypoint. CVPR, pp. 510 - 517, 2012.

[4] S.Lazebnik, C.Schmid, J.Ponce . Beyond Bags of Features:Spatial Pyramid Matching for Recognizing Natural Scene Categories.CVPR, pp.2169-2178,2006.

[5] K.Grauman and T.Darrell. Pyramid match kernels: Discriminative classification with sets of image features. ICCV, pp.1458-1465, 2005.

[6] L.Fei-Fei and P.Perona. A Bayesian hierarchical model for learning natural scene categories. CVPR, pp.524-531, 2005.

[7] Information on http: //www.vision.caltech.edu /Image_Datasets/Caltech101 .

[8] C.Harris and M.Stephens. A combined corner and edge detector. In Alvey vision conference, pp. 50-57, 1988.

[9] C.-C. Chang and C.-J. Lin. LIBSVM : a library for support vector machines. ACM Transactions on Intelligent Systems and Technology, pp.1-27, 2011

[10] J.Eichhorn and O.Chapelle. Object Categorization with SVM: Kernels for Local Features.Technical report,MPI for Biological Cybernetics, 2004. 